\title{
Well-Being and Education of Urology Residents During the COVID-19 Pandemic: Results of an American National Survey
}

\author{
Johnathan Khusid ${ }^{1}$, Corey Weinstein ${ }^{1}$, Adan Becerra ${ }^{2}$, Mahyar Kashani ${ }^{1}$, Dennis Robins ${ }^{1}$, \\ Lauren Fink ${ }^{1}$, Matthew Smith ${ }^{1}$, and Jeffrey Weiss ${ }^{1}$ \\ ${ }^{1}$ SUNY Downstate Health Sciences University \\ ${ }^{2}$ Affiliation not available
}

May 18, 2020

\begin{abstract}
Background: The rapid spread of COVID-19 has placed tremendous strain on the American healthcare system. Few prior studies have evaluated the well-being of or changes to training for American resident physicians during the COVID-19 pandemic. We aim to study predictors of trainee well-being and changes to clinical practice using an anonymous survey of American urology residents. Methods: An anonymous, voluntary, 47-question survey was sent to all ACGME-accredited urology programs in the United States. We executed a cross-sectional analysis evaluating risk factors of perception of anxiety and depression both at work and home and educational outcomes. Multiple linear regressions models were used to estimate beta coefficients and $95 \%$ confidence intervals. Results: Among approximately 1,800 urology residents in the USA, 356 (20\%) responded. Among these respondents, 24 had missing data leaving a sample size of 332. Important risk factors of mental health outcomes included perception of access to PPE, local COVID-19 severity, and perception of susceptible household members. Risk factors for declination of redeployment included current redeployment, having children, and concerns regarding ability to reach case minimums. Risk factors for concern of achieving operative autonomy included cancellation of elective cases and higher level of training. Conclusions: Several potential actions, which could be taken by urology residency program directors and hospital administration, may optimize urology resident well-being, morale, and education. These include advocating for adequate access to PPE, providing support at both the residency program and institutional levels, instituting telehealth education programs, and fostering a sense of shared responsibility of COVID-19 patients.
\end{abstract}

\section{What's already known about this topic?}

The COVID-19 pandemic has resulted in significant changes to the daily practice of health providers including resident physicians.

In some instances, physician specialists, have been redeployed to "front-line" services such as the medical wards, intensive care unit, and emergency room.

Despite the unique challenges presented by the pandemic, the impact on resident well-being, clinical practice, and education are largely unknown.

\section{What does this article add?}

This study is one of the first to assess resident physician wellness and education during the COVID-19 Pandemic.

We identified measures which may optimize urology resident well-being and education including advocating for adequate PPE, providing support at the residency program and institutional levels, instituting telehealth education programs, and fostering a sense of shared responsibility for COVID-19 patients. 


\section{INTRODUCTION}

In December 2019, a highly contagious novel coronavirus (SARS-CoV-2) was identified in Wuhan, China ${ }^{1}$, and on March $26^{\text {th }}, 2020$ the USA became the world's most afflicted nation with 81,321 cases of coronavirus disease 2019 (COVID-19) ${ }^{2,3}$. The rapid spread of COVID-19 has placed tremendous strain on the American healthcare system and prompted drastic steps to divert healthcare resources for the treatment of patients with COVID-19. For example, on March $14^{\text {th }}, 2020$ the Surgeon General advised all hospitals to halt elective surgery $^{4}$. Additionally, physicians have increasingly used telemedicine to facilitate social distancing ${ }^{5}$ and in some instances, physician specialists, have been redeployed to "frontline" services such as the medical wards, intensive care unit, and emergency room ${ }^{6}$.

In addition to clinical practice changes, numerous academic meetings have been cancelled, licensing exams are being rescheduled, and fellowship interviews are being conducted using teleconferencing software ${ }^{7}$. Beyond the educational and structural changes experienced, COVID-19 threatens the well-being of residents as nationwide personal protective equipment (PPE) shortages leave frontline workers at increased risk of viral exposure $^{8}$. Furthermore, a recent study conducted in Wuhan during the COVID-19 pandemic reported that frontline workers were at risk of unfavorable mental health outcomes ${ }^{9}$.

Despite these monumental changes and the unique challenges presented by the pandemic, the impact on resident well-being, clinical practice, and education are largely unknown. To address this gap, in the current study we aim to assess the well-being, clinical practice, and education of urology residents throughout the USA during the COVID-19 pandemic through the use of an anonymous survey. Given that routine urology practice encompasses elements of outpatient clinic, inpatient medicine, and surgery, and the low number of urology trainees nationally relative to other specialties, a national study of urology trainees may provide an important and timely initial assessment. To our knowledge, this is one of the first studies assessing trainees' well-being, clinical practice, and education during the COVID-19 pandemic.

\section{METHODS}

\section{Study Design}

The study obtained exempt status from the SUNY Downstate Health Sciences University Institutional Review Board. An anonymous, voluntary, 47-question survey was sent to all American Council for Graduate Medical Education (ACGME)-accredited urology programs in the USA by contacting each program's coordinator and/or director and each American Urologic Association (AUA) section secretary with the request to disseminate it to their residents. Additionally, social/professional networks were used to disseminate the survey, which was available from April 7, 2020 until April 11, 2020. The study is cross-sectional and assessed resident perceptions of personal, institutional and residency program responses to the pandemic. The survey utilized questions that were single-answer, multiple-answer, and Likert scales which were graded on a 1-5 scale with 1 representing "strongly disagree" and 5 representing "strongly agree."

\section{Outcomes}

The study evaluated six Likert scale outcomes related to resident mental health and training. Perceived severity of anxiety was evaluated using the following statements "I have increased anxiety at work due to the COVID-19 pandemic" and "I am more anxious outside of work due to the COVID-19 pandemic." Similar statements were used for perceived severity of depression: "I feel a sense of depression at work due to the COVID-19 pandemic." and "I feel a sense of depression outside of work due to the COVID-19 pandemic."

Declination of redeployment was measured with as follows: "I would decline redeployment to a COVID-19 service if given the option." Concern of operative autonomy was measured as follows: "I am concerned about my ability to operate independently as an attending urologist due to interruptions in training secondary to the COVID-19 pandemic."

\section{Risk Factors}

The objective was to identify independent risk factors of outcomes among urology residents during the 
pandemic. Potential risk factors included: resident age, gender identity, level of training, practice setting (urban/suburban/rural), AUA geographical section, perception of local COVID-19 severity (Likert), marital status, children, perceived household susceptibility to disease (Likert), history of COVID-19 symptoms, months of intensive care unit training, redeployment status, perceptions of availability of PPE (Likert) and COVID-19 testing, cancellation of elective cases, number of weekly operations before the pandemic, perceived program and hospital support (Likert), perception of shared responsibility with attendings (Likert), and perceived difficulty meeting case minimums (Likert).

\section{Statistical Analysis}

Data analysis was executed using R. Two-sided p-values with alpha=0.05 were used. Distributions of characteristics were tabulated using percentages for categorical variables and means with standard deviations for continuous variables. Six multivariable linear regressions were fit for the six outcomes using all risk factors as independent variables. Models estimated beta coefficients ( $\beta$ ) and $95 \%$ confidence intervals (CI) representing associations between risk factors and outcomes.

Linear regression assumptions were evaluated using plots and hypothesis tests. QQplots verified the assumption of normality. To test for heteroskedasticity, residual plots were generated along with a non-constant variance test. There was strong evidence of heteroskedasticity. To correct this, all outcomes employed a Box-Cox transformation. Lack of multicollinearity was confirmed by estimating variance inflation factors.

\section{RESULTS}

\section{Demographic Characteristics}

Among approximately 1,800 urology residents, 356 (20\%) responded. Among these respondents, 24 had missing data leaving a final sample size of 332. Table 1 reports the distributions of variables. The average age of the sample was 30.5. Of the 332 respondents, 117 (35\%) were female, $218(66 \%)$ were married, 262 (79\%) practiced in an urban setting, while $59(18 \%)$ practiced in a suburban setting. The most represented AUA regions were New York (32\%), Mid-Atlantic (15\%), and North-Central (14\%). A total of 72 (22\%) had been redeployed to a different service and 18 (5\%) reported a history of COVID19 symptoms.

\section{Risk Factors for Anxiety}

Figure 1 reports results of the risk factors associated with severity of anxiety outcomes. Perception of PPE availability was associated with lower severity of anxiety at work $(\beta=-0.14,95 \% \mathrm{CI}=-0.25,-0.02)$ and at home $(\beta=-0.13,95 \% \mathrm{CI}=-0.25,-0.01)$ whereas perception of local COVID-19 severity was associated with higher severity of anxiety at work $(\beta=0.21,95 \% \mathrm{CI}=0.09,0.33)$ and at home $(\beta=0.16,95 \% \mathrm{CI}=0.03$, $0.29)$. Perception of susceptible household member was associated with higher severity of anxiety at work $(\beta=0.14,95 \% \mathrm{CI}=0.05,0.23)$ and at home $(\beta=0.12,95 \% \mathrm{CI}=0.03,0.22)$. Urban practice setting $(\beta=0.69$, $95 \% \mathrm{CI}=0.02,1.36)$ and suburban practice setting $(\beta=0.71,95 \% \mathrm{CI}=0.01,1.42)$ was associated with higher anxiety severity at work compared to rural practice setting. Personal history of infection with COVID-19 was associated with higher severity of anxiety at work $(\beta=0.14,95 \% \mathrm{CI}=0.04,0.24)$. Amount of prior intensive care unit training was associated with lower severity of anxiety at work $(\beta=-0.08,95 \% \mathrm{CI}=-0.14,-0.02)$. Current redeployment was associated with higher severity of anxiety at work $(\beta=0.14,95 \% \mathrm{CI}=0.02,0.26)$ while perception of program support $(\beta=-0.11,95 \% \mathrm{CI}=-0.20,-0.03)$ was associated with lower severity of anxiety at work. Availability of testing if symptomatic was associated with lower severity of anxiety at home $(\beta=-0.39,95 \% \mathrm{CI}=-0.77,-0.02)$. Males reported lower severity of anxiety at work $(\beta=-0.3795 \% \mathrm{CI}=-0.63$, $-0.11)$ and at home $(\beta=-0.35,95 \% \mathrm{CI}=-0.63,-0.08)$.

\section{Risk Factors for Depression}

Figure 2 reports the results for depression outcomes. Perception of PPE availability was associated with lower severity of depression at work $(\beta=-0.22,95 \% \mathrm{CI}=-0.35,-0.09)$ and at home $(\beta=-0.22,95 \% \mathrm{CI}=-0.35,-0.09)$ whereas perception of local COVID-19 severity was associated with higher severity of depression at work $(\beta=0.17,95 \%$ CI $0.03,0.31)$. Perception of susceptible household member was associated with higher severity 
of depression at work $(\beta=0.14,95 \% \mathrm{CI}=0.05,0.23)$. Perception of program support $(\beta=-0.16,95 \% \mathrm{CI}=-$ $0.27,-0.05)$ was associated with lower severity of depression at work. Participation in $16-20(\beta=0.45,95 \%$ $\mathrm{CI}=0.23,0.68)$ operations/week prior to pandemic and $21+(\beta=0.67,95 \% \mathrm{CI}=0.01,1.323)$ operations/week compared to 0-5 operations/week before the pandemic was associated with higher depression severity at work. Males reported lower severity of depression at work $(\beta=-0.46,95 \% \mathrm{CI}=-0.75,-0.18)$ and at home $(\beta-0.42,95 \% \mathrm{CI}=-0.71,-0.13)$. Personal history of infection with COVID-19 was associated with higher severity of depression at home $(\beta=0.62,95 \% \mathrm{CI}=0.09,1.16)$. Concern of operative autonomy was associated with higher severity of depression at home $(\beta=0.12,95 \% \mathrm{CI}=0.01,0.23)$.

\section{Risk Factors for Declination of Redeployment and Concern of Operative Autonomy}

Figures 3 and 4 report the results for declination of redeployment and concern of operative autonomy, respectively. Perception of support from hospital administration $(\beta=-0.23,95 \% \mathrm{CI}=-0.40,-0.05)$ and shared responsibility between residents and attendings $(\beta=-0.22,95 \% \mathrm{CI}=-0.39,-0.07)$ were associated with lower declination of redeployment whereas concern regarding ability to reach graduation case requirements was associated with higher declination of redeployment $(\beta=0.16,95 \% \mathrm{CI}=0.00,0.32)$. Having children was associated with higher declination of redeployment $(\beta=0.80,95 \% \mathrm{CI}=0.36,1.24)$ whereas current redeployment was associated with lower declination of redeployment $(\beta=-0.72,95 \% \mathrm{CI}=-1.18,-0.25)$. Concern regarding ability to reach graduation case requirements was associated with higher concern of operative autonomy $(\beta=0.60,95 \% \mathrm{CI}=0.49,0.71)$. Cancellation of elective cases was associated with higher concern of operative autonomy $(\beta=0.74,95 \% \mathrm{CI}=0.20,1.28)$ while being married was protective $(\beta=-0.35,95 \% \mathrm{CI}=-0.62,-0.07)$. Residents in PGY4 $(\beta=0.53,95 \% \mathrm{CI}=0.31,1.04)$ and PGY5 $(\beta=0.80,95 \% \mathrm{CI}=0.31,1.30)$ had higher concern of operative autonomy.

\section{DISCUSSION}

The COVID-19 pandemic has placed significant strain on the American healthcare system. In response, major efforts have been made to divert healthcare resources for the treatment of COVID-19 patients. These include cancelling of elective surgery, redeployment of physician specialists to "frontline services," and the increased use of telemedicine. Additionally, in-person academic meetings and conferences have been cancelled to comply with social distancing recommendations. We sought to characterize urology resident education, clinical practice, and well-being with a national survey, and identified several important trends.

We identified several significant predictors of perceived anxiety and depression, both at work and home. Perceived adequacy of access to PPE was inversely related to all four mental health outcomes. That is, urology residents who reported adequate access to PPE reported lower levels of anxiety and depression. Similarly, a previous study of 1557 healthcare workers during the 2003 Severe Acute Respiratory Distress Syndrome pandemic found that lower stress levels were associated with PPE availability ${ }^{10}$.

The relationship between PPE availability and mental health during a pandemic may be related to fear of becoming ill and/or spreading the illness to loved ones. Indeed, urology residents who reported the presence of a household member (including themselves) who was susceptible to COVID-19 reported higher levels of anxiety at work, anxiety at home, and depression at work scores. This notion of self-protection is supported by a study of 169 healthcare workers during the avian flu epidemic in which $83 \%$ of respondents cited confidence in the hospital's ability to protect them as the most important factor influencing their willingness to report to work ${ }^{11}$. These findings suggest that ensuring adequacy of PPE availability is important for urology resident well-being during the COVID-19 pandemic.

Another potentially modifiable predictor of urology resident anxiety and depression was perception of support by the residency program. Residents who reported higher levels of program support had lower anxiety at work and depression at work scores. Furthermore, previous literature has described the importance of perceived support and appreciation by faculty in mitigating burnout amongst general surgery residents under regular circumstances ${ }^{12}$. Thus, it is important for program directors and faculty to regularly engage with residents and offer support and appreciation as this may improve well-being at work. 
Performing surgery is a key component of routine urology practice. However, with the onset of the pandemic, there has been a precipitous decline in operative volume with $94 \%$ of urology residents reporting that nononcologic cases have been cancelled and 37\% reporting that oncologic cases have been cancelled. The sharp decline is further illustrated by the decrease in percentage of residents reporting participation in 6 or more operations per week since the onset of the pandemic ( $89 \%$ vs. $5 \%$ ). This significant decrease in operative volume raises questions about disruption of surgical education. Urology residents tend to be the most active in the operating room during their senior and chief years and accordingly PGY-4 and PGY-5 residents reported higher levels of concerns regarding comfort with operative autonomy at the conclusion of training.

Routine urology practice also encompasses outpatient clinic visits. There has been a radical increase in the reported use of telehealth by urology services since the onset of the pandemic (10\% vs. $95 \%$ ). However, $82 \%$ of urology residents report that they have not been trained on how to perform effective telehealth visits. Given the reasonable possibility that increased telehealth usage will persist beyond the pandemic, urology residents would likely benefit from formal telehealth training.

Another major change to routine urology practice has been "redeployment" to a "frontline" COVID-19 service. Approximately one fifth of the urology residents surveyed have been redeployed, most commonly to the intensive care unit, medical wards, and emergency room. Of the redeployed residents, $77 \%$ report that their redeployment was mandatory. For all respondents, we assessed perception of declination of voluntary redeployment. Modifiable negative predictors of declination score were perception of institutional support and perception of shared responsibility for pandemic related activities with attendings. That is, urology residents who felt supported by their institution and that additional responsibilities were not being solely placed on the residents would be more likely to agree to voluntary redeployment. It may be helpful for hospital administrators to reach out to residents and inquire what resources they need to feel a greater sense of support (e.g. hazard pay, complementary lodging for self-quarantine, food subsidy). Additionally, responsibility for the care of COVID-19 patients should be shared between attendings and residents. Implementing these changes may improve morale by making redeployment feel more voluntary than mandatory.

Our study had several notable limitations. Our respondent rate was $20 \%$ and therefore not necessarily indicative of the entire population of urology residents. This may be an inherent limitation of using an optional survey in this population given that by comparison, the AUA-sanctioned resident survey conducted over three years from 2016-2018 had a respondent rate of only $26 \%{ }^{13}$. Additionally, the survey was predominantly distributed through secondary means (i.e. residency program directors and AUA section secretaries) rather than directly to respondents which may result in sampling error. Furthermore, a simple 1-5 scale was used for assessing depression and anxiety rather than a validated questionnaire such as the Patient Health Questionnaire 9. The use of a validated questionnaire may have provided more insight into the surveyed population. For example, in our study men reported lower depression and anxiety scores. Previous research has found that men tend to underreport anxiety and depression ${ }^{14,15}$. Without the use of a validated questionnaire, it is unclear if our findings are due to this known underreporting phenomenon or have another explanation.

Despite limitations, we have identified several important interventions which could potentially be undertaken by hospital administrators and urology programs to optimize urology resident well-being, education, and morale during the course of a pandemic. In summary these are: advocating for adequate access to PPE, providing support at both the residency program and institutional levels, instituting telehealth education programs, and fostering a sense of shared responsibility for COVID-19 patients. Interestingly, all of these findings are relatively general in nature and could potentially be applied to all specialties. Thus, we believe it is imperative to perform a follow up study across all specialties to assess the generalizability and validity of our findings. Furthermore, our study provides a unique and timely prospective, as it was conducted during a critical period of the pandemic in the US, capturing the days leading up to and including April $11^{\text {th }}, 2020$ (the date that the USA became the nation with the most total COVID-19 mortalities).

\section{Conclusions}


The COVID-19 pandemic has placed unprecedented strain on the healthcare system and prompted dramatic resource reallocation to minimize patient morbidity and mortality. These resource shifts have resulted in major changes to previous routines of urology residents. Our study has identified several potential actions that could be taken by residency programs and hospital administration which may optimize urology resident well-being, morale, and education. These include advocating for access to PPE, providing support at both the residency program and institutional levels, instituting telehealth education programs, and fostering a sense of shared responsibility for COVID-19 patients. Our study was limited in scope to urology residents. However, to our knowledge, ours was one of the first national study characterizing COVID-19 pandemic responses among American trainees. Importantly, these findings, if appropriately validated, could be applied to non-urology trainees. Thus, we recommend further research with a large national study of trainees from all specialties to assess the validity and generalizability of our findings.

\section{References}

1. Zhu N, Zhang D, Wang W, et al. A Novel Coronavirus from Patients with Pneumonia in China, 2019. N Engl J Med 2020;382:727-33.

2. The U.S. Now Leads the World in Confirmed Coronavirus Cases. The New York Times, 2020. (Accessed April 14, 2020, at https://www.nytimes.com/2020/03/26/health/usa-coronavirus-cases.html.)

3. COVID-19 Dashboard by the Center for Systems Science and Engineering (CSSE) at Johns Hopkins University (JHU). Johns Hopkins University, 2020. April 14, 2020, at https://coronavirus.jhu.edu/map.html.)

4. Hospitals Push Off Surgeries to Make Room for Coronavirus Patients. The Wall Street Journal, 2020. (Accessed April 14, 2020, at https://www.wsj.com/articles/hospitals-push-off-surgeries-to-make-room-forcoronavirus-patients-11584298575.)

5. Hospitals Use Telemedicine to Minimize Contact With Virus Patients. The Wall Street Journal, 2020. (Accessed April 14, 2020, at https://www.wsj.com/articles/hospitals-use-telemedicine-to-minimize-contactwith-virus-patients-11583337283.)

6. 'Today, We Are All Covid-19 Doctors'. The New York Times, 2020. (Accessed April 14, 2020, at https://www.nytimes.com/2020/04/06/opinion/coronavirus-doctor-covid.html.)

7. Khusid JA, Sullivan JF, Esdaille AR, Khargi R, Shields JM. Role of the Urologist During a Pandemic: Early Experience in Practicing on the Front Lines in Brooklyn, New York. Eur Uro In Press 2020.

8. Ranney ML, Griffeth V, Jha AK. Critical Supply Shortages - The Need for Ventilators and Personal Protective Equipment during the Covid-19 Pandemic. N Engl J Med 2020.

9. Lai J, Ma S, Wang Y, et al. Factors Associated With Mental Health Outcomes Among Health Care Workers Exposed to Coronavirus Disease 2019. JAMA network open 2020;3:e203976.

10. Maunder RG, Lancee WJ, Rourke S, et al. Factors associated with the psychological impact of severe acute respiratory syndrome on nurses and other hospital workers in Toronto. Psychosom Med 2004;66:938-42.

11. Irvin CB, Cindrich L, Patterson W, Southall A. Survey of hospital healthcare personnel response during a potential avian influenza pandemic: will they come to work? Prehospital and disaster medicine 2008;23:32835.

12. Appelbaum NP, Lee N, Amendola M, Dodson K, Kaplan B. Surgical Resident Burnout and Job Satisfaction: The Role of Workplace Climate and Perceived Support. The Journal of surgical research 2019;234:20-5.

13. 2018 The State of the Urology Workforce and Practice in the United States. American Urological Association, 2018. (Accessed April 14, 2020, at https://www.auanet.org/Documents/research/census/2018\%20The\%20State\%20of\%20the\%20Urology\%20Workforce\%20Cer

14. Addis ME. Gender and Depression in Men. Clinical Psychology: Science and Practice 2008;15:153-68. 
15. Levant RF. Toward the reconstruction of masculinity. Journal of Family Psychology 1992;5:379-402.

Tables/Figures

Table 1: Participant Characteristics

\begin{tabular}{|c|c|}
\hline Characteristic & Overall, $\mathrm{N}=332$ \\
\hline Age, mean $\pm \mathrm{SD}$ & $30.5 \pm 2.6$ \\
\hline Women, N (\%) & $117(35)$ \\
\hline \multicolumn{2}{|l|}{ Level of Training, N (\%) } \\
\hline PGY1 & $53(16 \%)$ \\
\hline PGY2 & $69(21 \%)$ \\
\hline PGY3 & $67(20 \%)$ \\
\hline PGY4 & $56(17 \%)$ \\
\hline PGY5 & $87(26 \%)$ \\
\hline \multicolumn{2}{|l|}{ Practice Setting, N (\%) } \\
\hline Urban & $262(79 \%)$ \\
\hline Suburban & $59(18 \%)$ \\
\hline Rural & $11(3 \%)$ \\
\hline \multicolumn{2}{|l|}{ AUA Geography, N (\%) } \\
\hline Western & $28(8 \%)$ \\
\hline South Central & $30(9 \%)$ \\
\hline North Central & $47(14 \%)$ \\
\hline Southeastern & $35(11 \%)$ \\
\hline Mid Atlantic & $49(15 \%)$ \\
\hline New York & $105(32 \%)$ \\
\hline New England & $22(6 \%)$ \\
\hline Declined & $16(5 \%)$ \\
\hline COVID19 Area Severity, mean \pm SD & $3.3 \pm 2.6$ \\
\hline Married, N (\%) & $218(66 \%)$ \\
\hline Children, N (\%) & $65(20 \%)$ \\
\hline Susceptible to COVID19, mean \pm SD & $2.1 \pm 1.4$ \\
\hline Ill with COVID19, N (\%) & $18(5 \%)$ \\
\hline Months of ICU Training, mean \pm SD & $1.4 \pm 1.1$ \\
\hline Redeployed, N (\%) & $72,(22 \%)$ \\
\hline Adequate Access to PPE, mean $\pm \mathrm{SD}$ & $3.4 \pm 1.1$ \\
\hline Asymptomatic Testing, N (\%) & $49(15 \%)$ \\
\hline Symptomatic Testing, N (\%) & $281(85 \%)$ \\
\hline Stopped Elective Cases, N (\%) & $312(94 \%)$ \\
\hline \multicolumn{2}{|l|}{$\begin{array}{l}\text { Number of Weekly Operations Pre-Pandemic, N } \\
(\%)\end{array}$} \\
\hline $0-5$ & $53(16 \%)$ \\
\hline $6-10$ & $69(21 \%)$ \\
\hline $11-15$ & $67(20 \%)$ \\
\hline $16-20$ & $56(17 \%)$ \\
\hline $21+$ & $87(26 \%)$ \\
\hline Support from Program, mean \pm SD & $4.1 \pm 1.0$ \\
\hline Support from Hospital, mean \pm SD & $3.2 \pm 1.2$ \\
\hline Shared Responsibility, mean \pm SD & $3.6 \pm 1.2$ \\
\hline Difficulty Meeting Case Minimums, mean \pm SD & $2.0 \pm 1.2$ \\
\hline Anxiety at Work, mean $\pm \mathrm{SD}$ & $3.8 \pm 1.1$ \\
\hline Anxiety at Home, mean \pm SD & $3.7 \pm 1.1$ \\
\hline
\end{tabular}




\begin{tabular}{ll}
\hline Characteristic & Overall, N=332 \\
\hline Depression at Work, mean \pm SD & $2.6 \pm 1.3$ \\
Depression at Home, mean \pm SD & $2.7 \pm 1.3$ \\
Declination of Redeployment, mean \pm SD & $2.7 \pm 1.5$ \\
Concern of Operative Autonomy, mean \pm SD & $2.3 \pm 1.3$ \\
\hline
\end{tabular}

\section{Hosted file}

Figure 1.docx available at https://authorea.com/users/322684/articles/451633-well-beingand-education-of-urology-residents-during-the-covid-19-pandemic-results-of-an-americannational-survey

\section{Hosted file}

Figure 2.docx available at https://authorea.com/users/322684/articles/451633-well-beingand-education-of-urology-residents-during-the-covid-19-pandemic-results-of-an-americannational-survey

\section{Hosted file}

Figure 3.docx available at https://authorea.com/users/322684/articles/451633-well-beingand-education-of-urology-residents-during-the-covid-19-pandemic-results-of-an-americannational-survey

\section{Hosted file}

Figure 4.docx available at https://authorea.com/users/322684/articles/451633-well-beingand-education-of-urology-residents-during-the-covid-19-pandemic-results-of-an-americannational-survey 